\title{
Research on Camera Calibration System Based on Binocular Stereo Vision
}

\author{
Chunyan Yang \\ College of Computer Science, Baicheng Normal University of Jilin Province, China \\ bcsyycy@163.com
}

Keywords: Camera calibration, 3D reconstruction, two-dimensional image, three-dimensional object

\begin{abstract}
Get the information of the three-dimensional object from the two-dimensional image, camera calibration is an essential and critical step. The position of a three-dimensional object, geometric information such as shape and state can be obtained from $2 \mathrm{D}$ image only by camera calibration and thus the information of 3D objects in real environment can be restored. The intensity of the reflected light of a point on a space object reflected on a two-dimensional image is the intensity of each bright spot on the image.
\end{abstract}

\section{Introduction}

The spatial geometry of the object is linked to each other by the position of the point on the picture. By camera calibration, we can calculate the corresponding relationship between the picture position and the corresponding spatial geometry position. The so-called camera calibration is to complete the calculation of fixed camera parameters through experiments. It can be said that the camera calibration process is the basis of the three-dimensional reconstruction process, the accuracy of camera calibration results is directly related to the accuracy of the subsequent 3D reconstruction and the reconstruction process of the subsequent object surface damage.

\section{Working Principle of CCD Camera.}

When the CCD camera is shooting, the spatial image information is imaging on the sensor of the CCD image through the lens. Optical image information is first converted to analog electrical signal when it is taken by CCD camera. It is then converted into a digital signal by an (A/D) converter. Then the digitized picture information is stored in the corresponding memory by the image processor, and finally it is stored in the computer through the digital interface. The working principle is shown in Figure. 1.

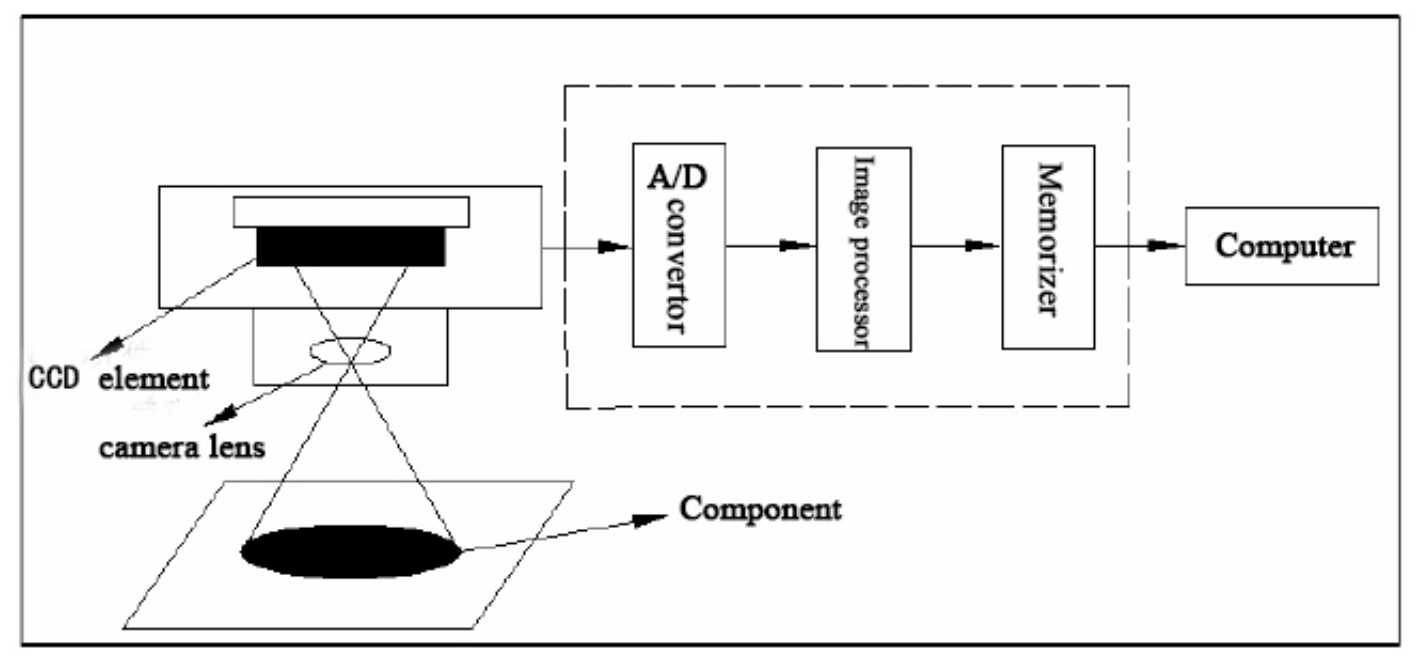

Fig.1 CCD camera schematic diagram 
Compared with the traditional camera technology, the image recorded by CCD camera can be reproduced directly and conveniently without complicated operation, such as developing and so on. Therefore, it greatly simplifies the follow-up processing of the camera work, and can easily and quickly display the captured picture through its own display screen.

\section{Camera Model.}

After the three-dimensional vision system obtains the image information through the camera, the geometric information such as the three-dimensional position and the shape characteristic of the object under test can be obtained by the calculation. The geometric information of objects in three-dimensional space is reflected in the two-dimensional image, which is each bright spot in the picture. The geometric relationship between a three-dimensional object and a two-dimensional photograph is determined by an optical imaging relationship. The camera model is the reproduction of the geometric relationship of optical imaging. The accuracy of the camera model directly affects the accuracy of the subsequent calibration results.

\subsection{Image Coordinate System, Camera Coordinate System and World Coordinate System.}

Before introducing the camera model, we introduce the three coordinate systems used in the camera model. Because in the process of image acquisition, the three-dimensional scene in the objective world is projected into the two-dimensional image of the camera. Therefore, the projection process can be used to describe and interpret the whole image changes. Each digital image is represented in the computer as an array of $\mathrm{M}^{*} \mathrm{~N}$, Therefore, the image captured by the camera is digitally processed and input into the computer system, and each set of values in the storage array corresponds to the brightness of the image point. Define the coordinate system as shown in Figure 2, the coordinate values of each pixel $(u, v)$ respectively represent the number of rows and columns of the pixel in the array so $(u, v)$ that is the coordinates of the image coordinate system in pixels.

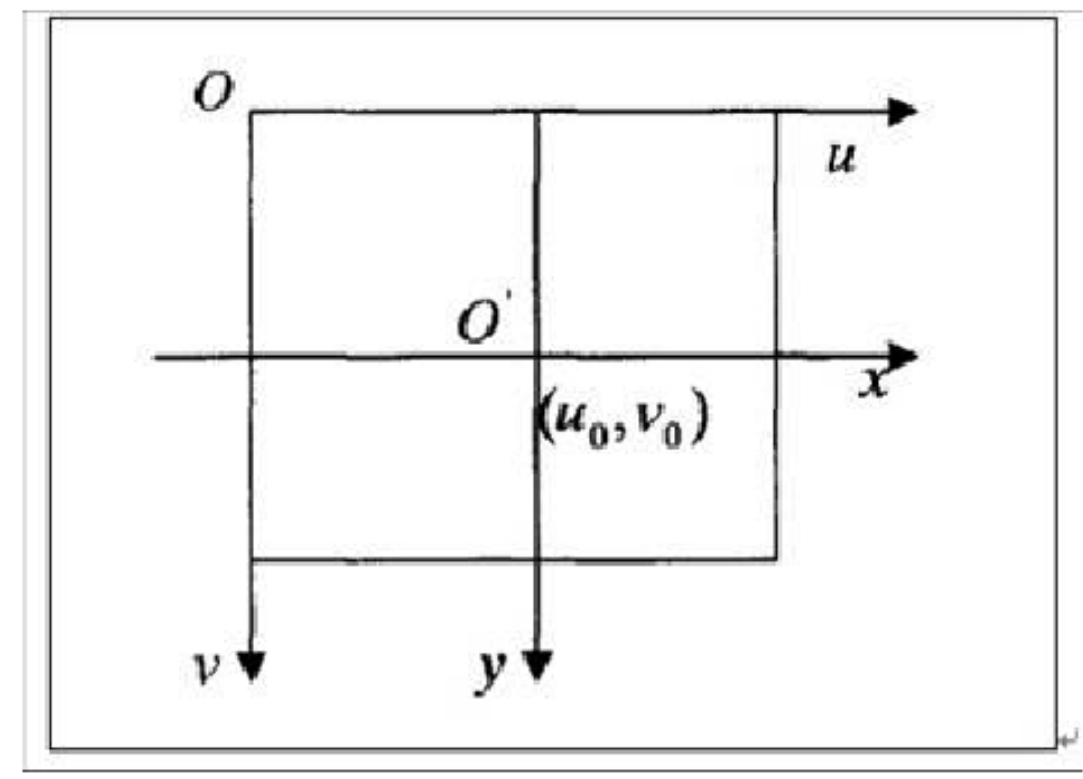

Fig. 2 CCD camera Coordinate

Due to the value of the coordinates represents only the number of columns and rows of the pixels in the image in the computer storage array. The actual positions of pixels in a two-dimensional image are not represented by physical units. Therefore, it is necessary to establish a new coordinate system to represent the position of its image points in the image, and generally select physical units to represent the coordinate system. As shown in Figure 3, the circle of the coordinate system is located at a point of one point in the image. $\mathrm{X}$ axis, $\mathrm{y}$ axis and image $\mathrm{U}$, respectively $\mathrm{V}$ is parallel, if the coordinate value of 0 in $\mathrm{U}-0-\mathrm{V}$ coordinate system is $\left(u_{0}, v_{0}\right)$, each pixel is on the axis, the 
physical dimensions in the direction of the $\mathrm{Y}$ axis can be expressed as $d x$ and $d y$ respectively. Then the coordinate relation of any pixel in the formula (1) image in the two coordinate systems can be expressed as follows:

$$
\begin{aligned}
& \mathrm{u}=\frac{x}{d_{x}}+u_{0} \\
& \mathrm{u}=\frac{y}{d_{y}}+v_{0}
\end{aligned}
$$

Expression (2) using homogeneous coordinates and matrix form

$$
\left[\begin{array}{l}
u \\
v \\
1
\end{array}\right]=\left[\begin{array}{ccc}
\frac{1}{d_{x}} & 0 & u_{0} \\
0 & \frac{1}{d_{y}} & v_{0} \\
0 & 0 & 1
\end{array}\right]\left[\begin{array}{l}
x \\
y \\
1
\end{array}\right]
$$

The camera aperture imaging geometry is shown in Figure 3 below. In the picture, $0_{\mathrm{C}}$ is called the center of light of the camera. $\mathrm{X}_{\mathrm{C}}$ of image coordinates, the $\mathrm{Y}_{\mathrm{C}}$ axis is parallel to the $\mathrm{X}$ and $\mathrm{Y}$ axes in the image plane respectively and the optical axis of the camera is perpendicular to the image plane. The focal point of the optical axis and the image plane is called the circle point of the image coordinate system. The coordinate system composed of $\mathrm{X}_{\mathrm{C}}, \mathrm{Y}_{\mathrm{C}}, \mathrm{Z}_{\mathrm{C}}$ and $\mathrm{0}_{\mathrm{C}}$ is called the camera coordinate system.

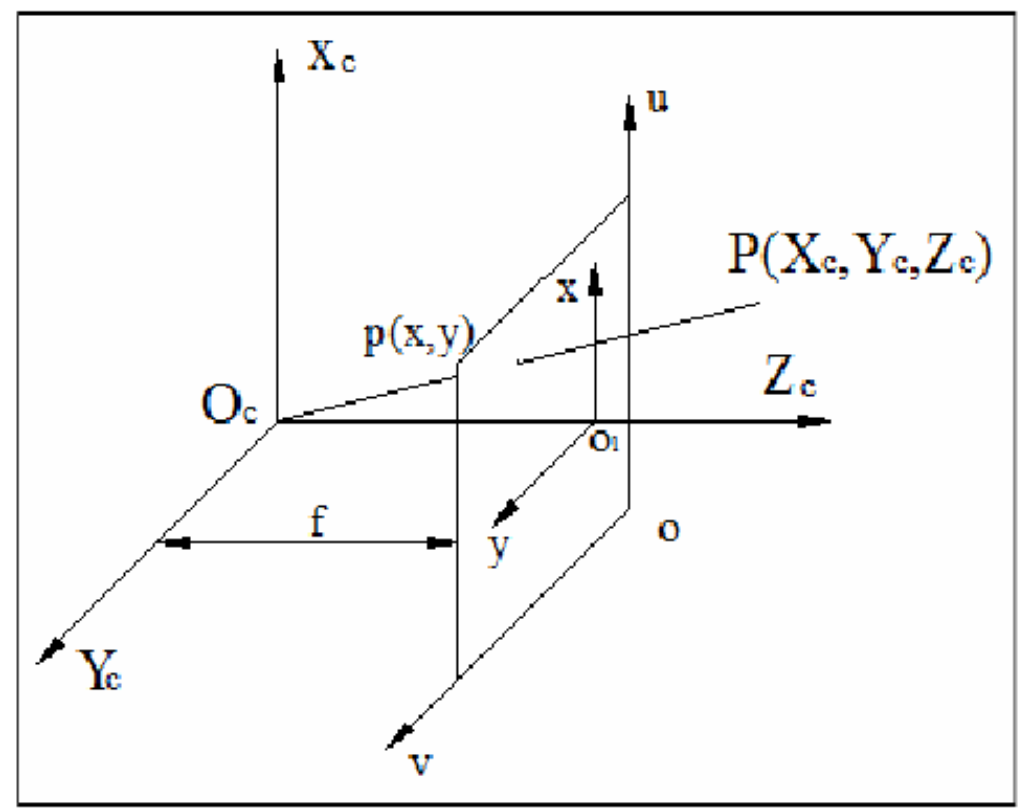

Fig 3 CCD camera image Coordinate

Because the camera can be placed anywhere in space, so if we need to select a new coordinate system in space to clearly describe the position of objects in space, the coordinate system is called the world coordinate system. The world coordinate system consists of $\mathrm{X}_{\mathrm{W}}, \mathrm{Y}_{\mathrm{W}}$ and $\mathrm{Z}_{\mathrm{W}}$ are composed of three variables. The relationship between world coordinate system and camera coordinate system can be expressed by rotation matrix $\mathrm{R}$ and translation vector $\mathrm{T}$. If $\left(\mathrm{X}_{\mathrm{W}}, \mathrm{Y}_{\mathrm{W}}, \mathrm{Z}_{\mathrm{W}}\right)$ and $(x, y, z)$ are used to represent the world coordinate system and camera coordinate system at any point in space, then the relationship between them is shown in formula (3).

$$
\left[\begin{array}{l}
x_{c} \\
y_{c} \\
z_{c}
\end{array}\right]=R\left[\begin{array}{l}
x_{w} \\
y_{w} \\
z_{w}
\end{array}\right]+t=\left[\begin{array}{lll}
r_{11} & r_{12} & r_{13} \\
r_{21} & r_{22} & r_{23} \\
r_{31} & r_{32} & r_{33}
\end{array}\right]\left[\begin{array}{l}
x_{w} \\
y_{w} \\
z_{w}
\end{array}\right]+t
$$

Homogeneous coordinates can be expressed as 


$$
\left[\begin{array}{l}
x \\
y \\
z \\
1
\end{array}\right]=\left[\begin{array}{ll}
R & t \\
0 & 1
\end{array}\right]\left[\begin{array}{c}
x_{w} \\
y_{w} \\
z_{w} \\
1
\end{array}\right]=M_{1}\left[\begin{array}{c}
x_{w} \\
y_{w} \\
z_{w} \\
1
\end{array}\right]
$$

Among them, $\mathrm{t}=\left[t_{x}, t_{y}, t_{z}\right]$ is called the world coordinate system origin, coordinates in the camera coordinate system, and the matrix $\mathrm{R}$ is the orthogonal matrix, and satisfies the following matrix conditions:

$$
\left\{\begin{array}{l}
r_{11}^{2}+r_{12}^{2}+r_{13}^{2}=1 \\
r_{21}^{2}+r_{22}^{2}+r_{23}^{2}=1 \\
r_{31}^{2}+r_{32}^{2}+r_{33}^{2}=1
\end{array}\right.
$$

Because the space position in the world coordinate system is determined by the variables of the unit orthogonal $t_{\mathrm{x}}, t_{\mathrm{y}}, t_{\mathrm{z}}$ these six parameters are called camera external parameters.

\subsection{Linear Model.}

Linear camera model is a more common and widely used camera model. That is the so-called pinhole camera imaging model the camera imaging geometric model reflects the image of each point of the position and space corresponding to the relationship between the point. Since the camera imaging lens distortion and other factors are not considered in the modeling process, the imaging process can be summarized as the following three steps:

The first step: from the world coordinate system to the camera coordinate system, the object will be converted $\left(X_{\mathrm{W}}, Y_{\mathrm{W}}, Z_{\mathrm{W}}\right)$ to $\left(X_{\mathrm{C}}, Y_{\mathrm{C}}, Z_{\mathrm{C}}\right)$, through the rotation matrix $\mathrm{R}$ and the translation vector T can be described as:

$$
\left[\begin{array}{l}
x_{c} \\
y_{c} \\
z_{c}
\end{array}\right]=R\left[\begin{array}{l}
x_{w} \\
y_{w} \\
z_{w}
\end{array}\right]+t
$$

The translation vector $\mathrm{T}=\left(t_{\mathrm{x}}, t_{\mathrm{y}}, t_{\mathrm{z}}\right)$ represents the coordinates of the origin of the world coordinate system in the camera coordinate system. The rotation matrix $R$ is represented by a $3 \times 3$-order unit orthogonal matrix.

The second step: the image coordinate system converts the camera coordinate system to a graph from $\left(X_{\mathrm{C}}, Y_{\mathrm{C}}, Z_{\mathrm{C}}\right)$ to $\left(X_{0}, Y_{0}\right)$. The geometric relationship between object point $P$ coordinate $\left(X_{\mathrm{C}}, Y_{\mathrm{C}}, Z_{\mathrm{C}}\right)$ in camera coordinate system and ideal image point $P_{0}\left(X_{0}, Y_{0}\right)$ in image coordinate system can be expressed by the following expression (7) the relation between object point $\mathrm{P}$ coordinate $\left(X_{\mathrm{C}}, Y_{\mathrm{C}}, Z_{\mathrm{C}}\right)$ and ideal image point $P_{0}\left(X_{0}, Y_{0}\right)$ in camera coordinate system.

$$
\left\{\begin{array}{l}
x_{0}=\frac{f}{z_{c}} x_{c} \\
y_{0}=\frac{f}{z_{c}} y_{c}
\end{array}\right.
$$

The homogeneous coordinates are expressed as

$$
\left[\begin{array}{l}
x_{0} \\
y_{0} \\
z_{0}
\end{array}\right]=\frac{1}{z_{c}}\left[\begin{array}{cccc}
f & 0 & 0 & 0 \\
0 & \mathrm{f} & 0 & 0 \\
0 & 0 & 1 & 0
\end{array}\right]\left[\begin{array}{c}
x_{c} \\
y_{c} \\
z_{c} \\
1
\end{array}\right]
$$

Step 3: convert the image coordinate system to the pixel coordinate system, convert the point $\left(X_{0}, Y_{0}\right)$ to the pixel coordinate $(u, v)$. The corresponding relation can be expressed in the following way:

$$
\left\{\begin{array}{l}
u=\frac{x}{d_{x}}+u_{0} \\
v=\frac{y}{d_{y}}+v_{0}
\end{array}\right.
$$

The physical dimensions of each pixel in the axis and $Y$ direction are represented by $d x, d y$. 


\subsection{Nonlinear Model.}

Because of the inevitable defects in the camera lens and the CCD photographic surface, large image distortion is likely to occur far away from the image center when using wide-angle camera for high-precision measurement. It is impossible to accurately describe the geometric relationship of imaging by using linear model only. As shown in Figure 4, there is a slight deviation between the line of $\mathrm{P}$ and 0 of the object square point and the point of intersection of the image plane and the original image point. This deviation is referred to as lens distortion in the camera process.

There are two main types of distortion: radial distortion and tangential distortion. The change of the radial curvature of the optical lens is the main factor causing the radial distortion. This distortion will cause the image point to move in the radial direction, so the farther away from the center of the image, the more obvious the deformation will be. Expressed as formula (10) by mathematical formula.

$$
\begin{aligned}
& \hat{x}=x+x\left[k_{1}\left(x^{2}+y^{2}\right)+k_{2}\left(x^{2}+y^{2}\right)^{2}\right] \\
& \hat{y}=y+y\left[k_{1}\left(x^{2}+y^{2}\right)+k_{2}\left(x^{2}+y^{2}\right)^{2}\right]
\end{aligned}
$$

The upper middle coordinate value $(x, y)$ is the ideal imaging point coordinate based on the linear camera model while $(x, y)$ is the actual imaging point coordinate value after distortion. $k_{1}, k_{2}$ is called distortion coefficient. If it is necessary to consider the effect of tangential distortion on camera calibration, then the calibration process requires the use of a nonlinear optimization algorithm. But in this process, the stability of the system will change with the introduction of too many nonlinear parameters, so the tangential distortion is usually ignored.

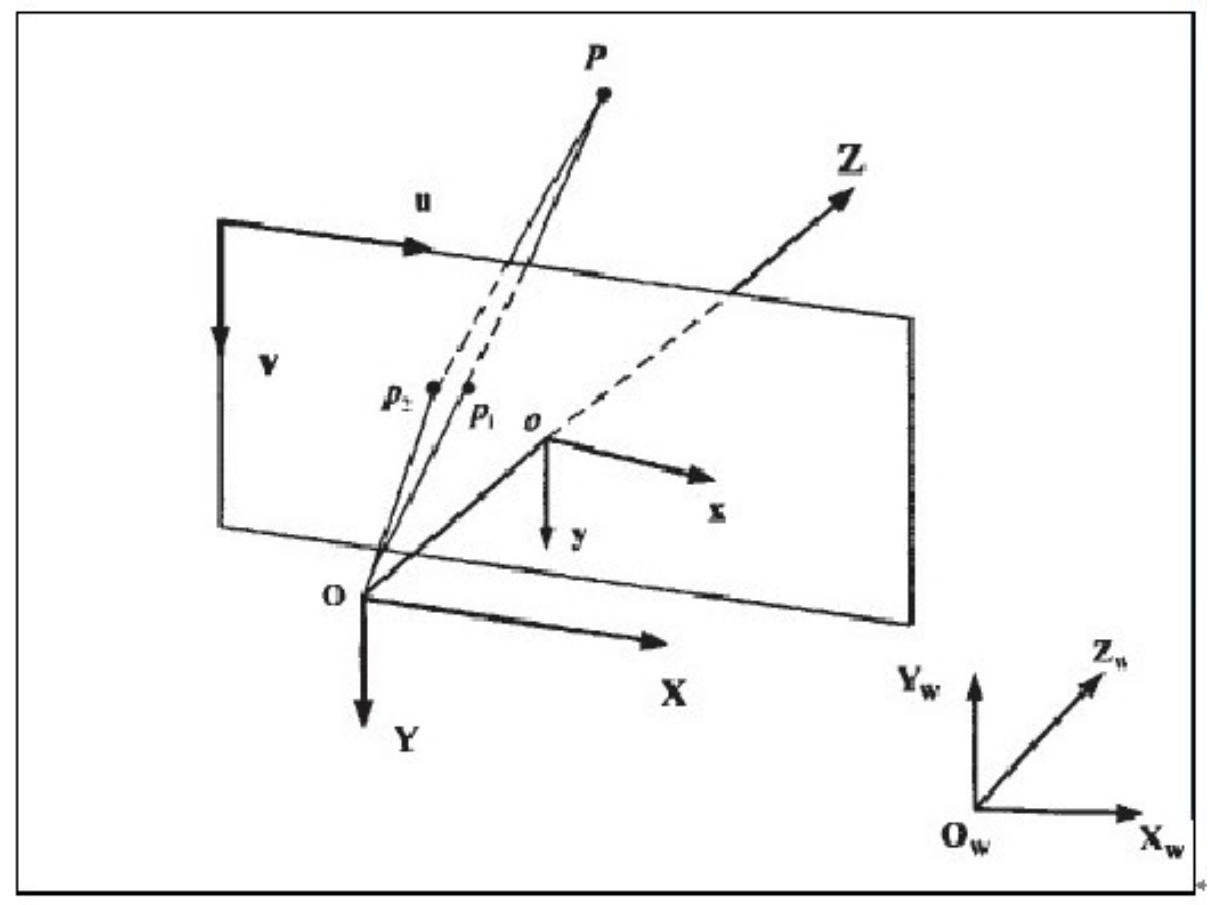

Fig.4 non-linear camera model

\section{Summary}

Camera calibration is an important part of binocular stereo vision. Whether the calibration results are accurate or not directly affects the extraction and damage calculation of the concave-convex depth of the back object surface. This paper first introduces the camera calibration process used in the image coordinate system camera coordinate system and world coordinate system followed by a detailed description of the camera calibration methods. Finally, according to the actual test process, the camera with fixed position is calibrated, the corresponding internal and external parameters are obtained, and the experimental results are given. 


\section{References}

[1] Guo Li-Li, Study on camera calibration and ranging in traffic accident scene [D]. Shandong Normal University, 2009.

[2] Li Hongyan. Calculation of central image coordinates based on geometric invariance of projective transformation [J]. Computer and modernization, 2010 09:41-46.

[3] Liu Dechao. Research on pavement crack recognition based on digital image processing [D]. Changsha University of technology. 2009.

[4] Li Jin-hui Image processing to detect cracks in highway pavement [J]. Journal of Chang'an University (natural science 2004.05, 24 (3): 24-29.)

[5] Xu Juan. A non-local method for image denoising [D]. Nanjing University of technology, 2009.

[6] He Junfeng. Research on image edge detection operator based on visual principle [D]. Huazhong University of science and technology, 2006. 